EXTENDED REPORT

\title{
A multicentre report from the Mexican Retinoblastoma Group
}

\section{Leal-Leal, M Flores-Rojo, A Medina-Sansón, F Cerecedo-Díaz, S Sánchez-Félix, O González- Ramella, F Pérez-Pérez, R Gómez-Martínez, A Quero-Hernández, E Altamirano-Álvarez, F Alejo- González, J Figueroa-Carbajal, A Ellis-Irigoyen, I Tejocote-Romero, R Cervantes-Paz, F Pantoja- Guillén, L Vega-Vega, F Carrete-Ramírez}

Br J Ophthalmol 2004;88:1074-1077. doi: 10.1136/bjo.2003.035642
See end of article for
authors' affiliations .....................

Correspondence to: Dr C Leal-Leal, Insurgentes Sur 3700-c Mexico 04530 Mexico City, Mexico; drcarlosaleal@msn.com

Accepted 7 January 2004
Background: Retinoblastoma (RB) is a relatively uncommon tumour in childhood. The incidence of retinoblastoma in Mexico is probably higher than the incidence reported worldwide, however there is not enough information about the characteristics of this illness in Mexico. This report aims to present the results of a multicentre clinical survey of RB in Mexico.

Methods: A retrospective study was carried out on all RB cases treated in 16 institutions during the last six years. The variables analysed were age at diagnosis, sex, affected eyes, treatment modalities, and pathological staging. Overall survival was obtained.

Results: The authors analysed 500 cases; age range was 0-182 months. There were 364 unilateral cases (72.8\%). Enucleation was performed in $84.9 \%$ of the patients. The St Jude's staging was: $7.4 \%$ stage I, $52.8 \%$ stage II, $18.0 \%$ stage III, $11.4 \%$ stage IV, $7.2 \%$ not evaluated, and $3.2 \%$ missing data. Chemotherapy was used in $74.4 \%$ of the patients. Disease free survival was $89 \%$ at 73 months follow up. Conclusions: The paper presents a great number of cases and pioneers multicentre studies in paediatric ophthalmology and oncology in this country. Given the great number of patients in advanced stages and the variability on treatment schemes, it is evident that it is mandatory to work in a cooperative group and develop a national early detection programme as well as a treatment protocol which include all specialists involved in the care of patients with RB.
$\mathrm{R}$ etinoblastoma $(\mathrm{RB})$ is the most common malignant ocular tumour in childhood. ${ }^{1}$ In the United States the incidence is 11 new cases of RB per million population under 5 years of age. ${ }^{2}$ In developing countries, most of the cases are detected at advanced stages. ${ }^{3-5}$ At the present time, nearly all patients diagnosed during the early stages can achieve a prolonged disease free survival, ${ }^{6}$ and at least $50 \%$ of the affected eyes can be preserved. ${ }^{7}$ In Mexico, RB may represent the second most frequent malignant solid tumour in paediatrics. ${ }^{8}$ However, there is no accurate information regarding the frequency and clinical characteristics of RB in the country. In a previous study we reported a series of 552 patients from a single institution. .' The study included patients diagnosed over a period of 15 years, with a minimum follow up of 4 years. The institution studied was a national reference hospital; most of those patients were from the southeast and central parts of the country, so the results presented in that report may not represent the clinical characteristics of retinoblastoma in Mexico.

The purpose of this paper is to present the results of a multicentre study on the frequency and clinical characteristics of RB in Mexican children around the country.

\section{MATERIALS AND METHODS}

A multicentred, retrospective, and descriptive study was carried out. Sixteen Mexican centres provided information on patients with RB diagnosed between 1 January 1997 and 31 December 2002. The contributing centres were as follows (number of patients shown in brackets): Centro Estatal de Cancerología de Jalapa (4); Centro Médico de Occidente, IMSS Jalisco (13); Centro Médico La Raza (41); Centro Médico Nacional SXXI (43); Hospital Civil de Durango (2); Hospital Civil de Guadalajara (24); Hospital del Niño
Morelense (10); Hospital del Niño Oaxaqueño (13); Hospital General de México (21); Hospital Pediátrico de Sinaloa (12); Hospital Infantil de México Federico Gómez (54); Hospital Central Universitario de San Luis Potosí (12); Hospital del Niño de Villahermosa (9); Instituto Nacional de Pediatría (229); Instituto Materno Infantil de Estado de México (7), and Hospital O’Horan de Mérida (6).

Recorded data included age at diagnosis, date of diagnosis, ocular affection (unilateral or bilateral) at diagnosis, stage at diagnosis according to St Jude's staging system, ${ }^{10}$ treatment modalities used (radiotherapy, surgery, and chemotherapy), and date of last visit. The survey did not consider information regarding second malignancies and treatment related deaths. The status of the patient evaluated by clinical examination and/or radiological studies at last visit was considered to be: alive with no evidence of disease; alive with active disease (patients with neoadyuvancy were considered in this group); dead with no evidence of disease, and dead with active disease.

Statistical analyses were performed using the SPSS system (SPSS for Windows 10.0; SPSS Inc, Chicago, IL, USA). Descriptive statistics for each variable were obtained. The Kaplan-Meier method with log rank test was used to determine overall survival. Differences between groups were considered significant when two sided $\mathrm{p}$ value was $<0.5$.

\section{RESULTS}

Between January 1997 and December 2002, a total of 500 patients were diagnosed with RB in the participating centres (mean 83.3 new cases per year). Figure 1 presents the number of patients per year; 262 were male and 238 female

Abbreviations: $R B$, retinoblastoma. 


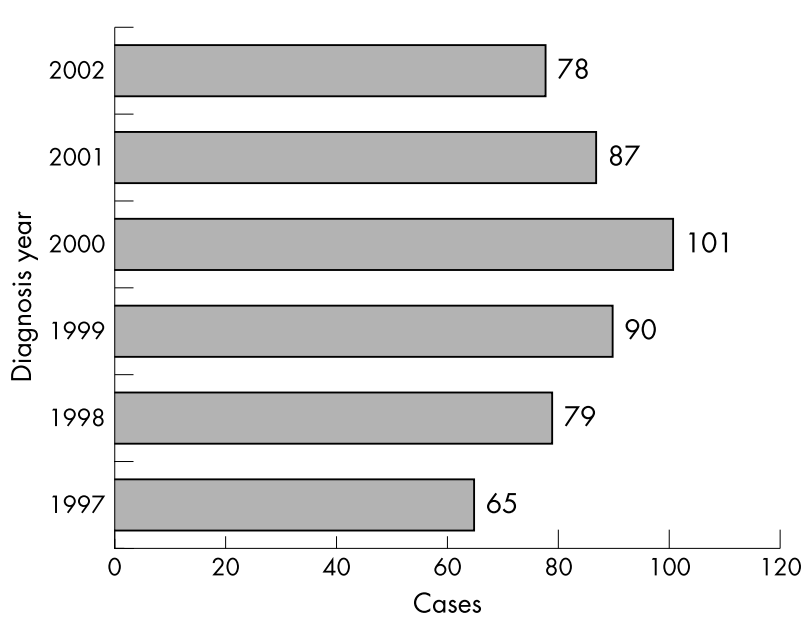

Figure 1 Frequency of new patients per year.

(M:F ratio 1.1:1.0). The age at diagnosis ranged from 1 day to 182 months, with a mean of 27.68 months (median 24). Initial ocular presentation was unilateral disease in 364 $(72.8 \%)$ and bilateral disease in $136(27.20 \%)$ patients. Mean age at diagnosis for unilateral cases was 30.96 months (median 28), whereas mean age for bilateral cases was of 18.87 (median 14).

Most of the centres did not provide information concerning the ocular stage at diagnosis (Reese-Elsworth staging system); therefore this data is not included in the report. Stage at diagnosis (St Jude's staging system) is presented in table 1, showing that almost $30 \%$ of the patients were diagnosed at advanced stages. Approximately $13 \%$ of the cases could not be staged due to multiple causes such as neoadyuvancy, incorrect management of the enucleated eye, or insufficient material of the optic nerve.

\section{Treatment}

The treatment modalities were mainly surgery, chemotherapy, and radiotherapy. Enucleation was performed in $87.62 \%$ of the patients (table 2). External radiotherapy was used in 131 patients $(26.2 \%)$; the indications for this treatment modality were adjuvant therapy in orbital or metastatic disease and eye rescue for bilateral disease when vitreous seedlings were present. The stage at diagnosis for radiated patients is presented in table 3. Among radiated patients 57 were bilateral. Seventy nine patients with bilateral disease were not radiated because the preserved eye had an early ocular stage and did not need this modality.

Chemotherapy was applied in $372(74.4 \%)$ patients. The indications for chemotherapy were: in 37 patients neoadyuvancy for eye preservation; adyuvant chemotherapy in 147

\begin{tabular}{|c|c|c|c|c|c|c|}
\hline \multirow[b]{2}{*}{ St Jude's stage } & \multicolumn{2}{|l|}{ Unilateral } & \multicolumn{2}{|l|}{ Bilateral } & \multicolumn{2}{|l|}{ Total } \\
\hline & Frequency & $\%$ & Frequency & $\%$ & Frequency & $\%$ \\
\hline I Limited to retino & a 22 & 6.0 & 15 & 11.0 & 37 & 7.4 \\
\hline $\begin{array}{l}\text { II Limited to the } \\
\text { eye }\end{array}$ & 194 & 53.3 & 70 & 51.5 & 264 & 52.8 \\
\hline $\begin{array}{l}\text { III Limited to the } \\
\text { orbit }\end{array}$ & 75 & 20.6 & 15 & 11.0 & 90 & 18.0 \\
\hline $\begin{array}{l}\text { IV Metastatic } \\
\text { disease }\end{array}$ & 44 & 12.1 & 13 & 9.6 & 57 & 11.4 \\
\hline Not classifiable & 19 & 5.2 & 17 & 12.5 & 36 & 7.2 \\
\hline Missing data & 10 & 2.8 & 6 & 4.4 & 16 & 3.2 \\
\hline Total & 364 & 100.0 & 136 & 100.0 & 500 & 100.0 \\
\hline
\end{tabular}

Table 2 Surgery modalities used

\begin{tabular}{lrrrr}
\hline & Unilateral & $\%$ & Bilateral & \multicolumn{1}{c}{$\%$} \\
\hline No surgery & 5 & 1.4 & 9 & 6.6 \\
$\quad$ Rejects surgery & 9 & 2.5 & 14 & 10.3 \\
$\quad$ Eye preservation & 330 & 90.6 & 97 & 71.3 \\
Unilateral enucleation & 0 & 0.0 & 11 & 8.1 \\
Bilateral enucleation & 17 & 4.7 & 5 & 3.7 \\
Unilateral exenteration & 3 & 0.8 & 0 & 0.0 \\
Missing data & 364 & 100.0 & 136 & 100.0 \\
Total & & & & \\
\hline & & & & \\
\end{tabular}

patients in advanced stages (orbital and metastatic disease), and 188 patients with ocular disease (in many centres their chemotherapy protocol still considers its use necessary). More than 15 different regimens were used by the participating centres (table 4). Twenty one per cent (106) of the patients received chemotherapy based on cyclophosphamide and doxorrubicyn, 50.6\% (253) received chemotherapy based on cisplatin or carboplatin plus other drugs, and $2.6 \%$ (13) received treatment based on research/ new drugs protocols or miscellaneous schemas. Twenty three per cent of the patients did not receive chemotherapy agents.

The calculated overall survival was $85 \%$ at 73 months of follow up; the mean survival time was 23.1 months (median 19.4). Figure 2 presents the overall survival by stage.

\section{DISCUSSION}

In Mexico, retinoblastoma is the second most frequent solid malignancy in paediatric patients, the most frequent being central nervous system tumours. ${ }^{11}$ Several centres that treat $\mathrm{RB}$ in Mexico did not participate in this survey so the number of cases of RB in Mexico could be higher than reported here. Hurwitz has suggested that the number of new cases per year could be higher in developing countries than in developed. ${ }^{12}$ However, with the data collected thus far we could not support this hypothesis.

As mentioned above, $45.8 \%$ of the patients were treated in a single institution (Instituto Nacional de Pediatría). This situation could be related to many factors: firstly, it is a national reference centre; secondly, for many years it was one of the few centres in the country for the treatment of retinoblastoma, and even now that other centres are working, ophthalmologists and general practitioners do not refer patients to other centres; finally, because many non-profit organisations support treatment in this centre but not in others. One of the aims of the retinoblastoma group is to create a national reference programme to better distribute the medical attention for RB patients, and reduce the risk of lost follow up by minimising the costs of transportation to the treatment centres.

The mean age at diagnosis was similar to what we reported in our previous study, ${ }^{9}$ and higher than that reported in other series from developed countries. ${ }^{13}$ Diagnosis at older ages seems to be a common finding in developing countries. ${ }^{14}$ In

Table 3 Radiated patients: stage at diagnosis

\begin{tabular}{lcc}
\hline St Jude's stage & Frequency & $\%$ \\
\hline I Limited to retina & 4 & 3.05 \\
II Limited to the eye & 31 & 23.67 \\
III Limited to the orbit & 47 & 35.88 \\
IV Metastatic disease & 25 & 19.08 \\
Not classifiable & 17 & 12.98 \\
Missing data & 7 & 5.34 \\
Total & 131 & 100.0 \\
\hline
\end{tabular}




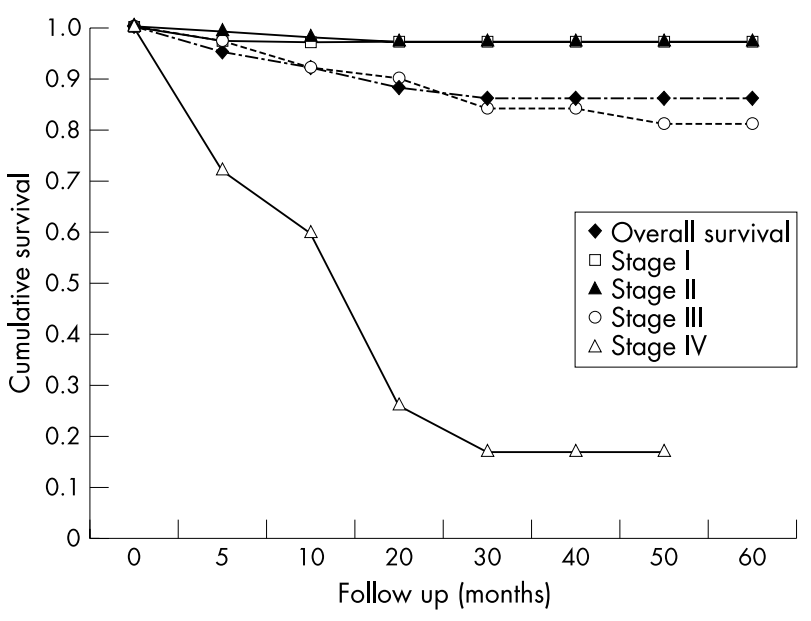

Figure 2 Overall survival by St Jude's stage (log rank 188.9, $\mathrm{df}=4$, significance 0.00001 ).

our previous study about $47 \%$ of the patients presented in advanced stages; in contrast, in the present report only one third of the patients were diagnosed at advanced stages ( St Jude's stages III and IV). In any case, these data suggest the need to develop a national early detection programme. Such a programme would permit us to decrease the number of patients with advanced extra ocular disease and offer less aggressive treatments at lower costs.

The distribution by affected eye is similar to our previous report and other international reports. ${ }^{14}$ Unfortunately, we do not have reliable data concerning the kind of community (rural or urban) where the patients come from, but this issue should be evaluated in a prospective study as there are reports of a higher prevalence of the disease in rural communities. ${ }^{15}$

Chemotherapy regimens used reflect the evolution of treatment of RB. Some centres are still using the regimens proposed by the Paediatric Oncology Group in $1972^{10}{ }^{16}$; other centres have modified their treatments to include drugs such as carboplatin plus VP 16, and in a few centres new drugs such as taxol, irinotecan, and other new agents are being tested in metastatic disease. Even the indications for chemotherapy vary among centres; in some of them chemotherapy is still used for patients in stage II. One of the aims of the Mexican Retinoblastoma Group is the

\begin{tabular}{|lrr|}
\hline \multicolumn{3}{|c|}{ Table 4 Chemotherapy: drugs schema used } \\
\hline \multicolumn{2}{|c|}{ Frequency $\%$} \\
\hline VCR-CFX & 28 & 5.6 \\
VCR-ADR & 4 & 0.8 \\
VCR+CFX+ADR & 72 & 14.4 \\
VP16+CFX & 2 & 0.4 \\
CDDP+VP16+CFX & 7 & 1.4 \\
Carboplatin+VP16+CFX & 54 & 10.8 \\
Carboplatin+VP16+VCR & 20 & 4.0 \\
Carboplatin+VP16 & 129 & 25.8 \\
CDDP+VP16 & 10 & 2.0 \\
Carboplatin & 16 & 3.2 \\
Carboplatin+VCR & 5 & 1.0 \\
Carboplatin+VP16 Altering VCR-CFX & 9 & 1.8 \\
ICE & 3 & 0.6 \\
Taxol & 2 & 0.4 \\
lrinotecan & 3 & 0.6 \\
Miscellaneous & 8 & 1.6 \\
No chemotherapy & 116 & 23.2 \\
Missing data & 12 & 2.4 \\
Total & 500 & 100.0 \\
\hline & & \\
\hline
\end{tabular}

development of a national treatment protocol, which would enhance the medical care of these patients and would open the possibility to perform controlled clinical trials.

The survival of this series of patients is similar to other large series reported before ${ }^{13}{ }^{16}$ nevertheless the follow up is still short. In comparison with our previous report, this series presents higher survival rates for stages II and III. Schvartzman et al ${ }^{16}$ from Buenos Aires, reported similar overall survival rates (84\%). Antoneli et al ${ }^{17}$ from Sao Paolo, and Chantada et $a l^{18}$ from Buenos Aires, report similar survival rates for orbital and metastatic disease. The common finding in all these series is the low survival rates for metastatic disease. As described above, the survival of our patients is similar to other series from Latin America; however the comparison between them is difficult as our follow up period is still short and the reports mentioned are from single institutions with more standardised treatments, whereas our report is a multicentre national study with a great diversity of chemotherapeutic regimens.

Given the number of new cases per year registered in this survey, RB is a frequent neoplasia in Mexico. It is necessary to create a national registry to understand the actual impact of RB in our country. The late diagnosis is a common problem in developing countries, and should be controlled to enhance prognosis and quality of life for these patients. In the same way, treatments need to be standardised so that every centre can offer the best available treatments at the lowest cost for patients. There is a lot of work to be done; nevertheless survival for RB patients in Mexico is similar to what has been reported in other series. ${ }^{16-24}$

This survey is relevant not just because of the data obtained, but because is the first attempt to work in a cooperative multicentre group in paediatric oncology in Mexico.

\section{Authors' affiliations}

C Leal-Leal, M Flores-Rojo, Instituto Nacional de Pediatría, DF, Mexico A Medina-Sansón, Hospital Infantil de México Federico Gómez, DF, Mexico

F Cerecedo-Díaz, Centro Médico Nacional SXXI, DF, Mexico

S Sánchez-Félix, Centro Médico La Raza, DF, Mexico

O González-Ramella, Hospital Civil de Guadalajara, Jalisco, Mexico F Pérez-Pérez, Hospital General de México, DF, Mexico

R Gómez-Martínez, Centro Médico de Occidente IMSS, Jalisco, Mexico

A Quero-Hernández, Hospital del Niño Oaxaqueño, Oaxaca, Mexico

E Altamirano-Álvarez, Hospital Pediátrico de Sinaloa, Sinaloa, Mexico F Alejo-González, Hospital Central de San Luis Potosí, San Luis Potosí, Mexico

J Figueroa-Carbajal, Hospital del Niño Morelense, Morelos, Mexico A Ellis-Irigoyen, Hospital del Niño de Villahermosa, Tabasco, Mexico I Tejocote-Romero, R Cervantes-Paz, Instituto Materno Infantil de Estado de México, Mexico

F Pantoja-Guillén, Hospital O'Horan de Mérida, Yucatan, Mexico

L Vega-Vega, Centro Estatal de Cancerología de Jalapa, Veracruz, Mexico

F Carrete-Ramírez, Hospital General de Durango, Durango, Mexico

\section{REFERENCES}

1 Grabowski EF, Abramson DH. Intraocular and extraocular retinoblastoma. Hematol Oncol Clin North Am 1987;1:721-35.

2 Devesa S. The incidence of retinoblastoma. Am J Ophthalmol 1975:80:263-5.

3 Sahu S, Banavali SD, Pai SK, et al. Retinoblastoma: problems and perspectives from India. Pediatr Hematol Oncol 1998;15:501-8.

4 Ajaiyeoba IA, Akang EE, Campbell OB, et al. Retinoblastomas in Ibadan: treatment and prognosis. West Afr J Med 1993; 12:223-7.

5 Zygulska-Mach H, Krukar-Baster K, Sajak-Hydzik K. Preliminary results of observing 18 cases of retinoblastoma carried within the international research program RICS. Klin Oczna 1994;96:21-3.

6 Rodriguez-Galindo C, Wilson MW, Haik BG, et al. Treatment of intraocular retinoblastoma with vincristine and carboplatin. J Clin Oncol 2003;21:2019-25. 
7 Lumbroso L, Doz F, Levy C, et al. Diode laser thermotherapy and chemothermotherapy in the treatment of retinoblastoma. J Fr Ophtalmol 2003;26:154-9.

8 Rivera-Luna R. Los problemas de la Hemato-Oncología Pediatrica en México. Bol Hosp Inf Mex 2003;60:125-31.

9 Leal-Leal C, Rivera-Luna R, Tovar-Guzmán V, et al. Risk of dying of retinoblastoma in Mexican children, Med Pediatr Oncol 2002;38:211-13.

10 Pratt CB. Management of malignant solid tumors in children. Pediatr Clin North Am 1972;19:1141-55.

11 Registro Histopatólogico de Neoplasias Malignas en México, 1993-1996 DGE-SSA, 2000, México.

12 Hurwitz RL, Shields CL, Shields JA, et al. Retinoblastoma. In: Pizzo PA, Poplack DG, eds. Principles and practice of pediatric oncology, 4th ed. Philadelphia, USA: Lippincott Williams \& Wilkins Publisher, 2002:825-46.

13 Khelfaoui F, Validire P, Auperin A, et al. Histopathologic risk factors in retinoblastoma: a retrospective study of 172 patients treated in a single institution. Cancer 1996;77:1206-13.

14 Gunalp I, Gunduz K, Arslan Y. Retinoblastoma in Turkey: diagnosis and clinical characteristics. Ophthalmic Genet 1996;17:21-7.

15 Schultz KR, Ranade S, Neglia JP, et al. An increased relative frequency of retinoblastoma at a rural regional referral hospital in Miraj, Maharashtra, India. Cancer 1993;72:282-6.
16 Schvartzman E, Chantada G, Fandiño A, et al. Results of a stage-based protocol for the treatment of retinoblastoma. J Clin Oncol 1996; 14:1532-6

17 Antoneli CB, Steinhorst F, de Cassia Braga Ribeiro K, et al. Extraocular retinoblastoma: a 13-year experience. Cancer 2003;98:1292-8.

18 Chantada G, Fandino A, Casak S, et al. Treatment of overt extraocular retinoblastoma. Med Pediatr Oncol 2003;40:158-61.

19 Kao LY, Su WW, Lin YW. Retinoblastoma in Taiwan: survival and clinical characteristics 1978-2000. Jpn J Ophthalmol 2002;46:577-80.

20 Bouguila H, Malek I, Boujemaa C, et al. Prognosis of retinoblastoma. Report of 50 cases. J Fr Ophtalmol 2001;24:1053-6.

21 Magnani C, Pannelli F, Mosciatti P, et al. Survival analysis of childhood tumours: data from the Italian cancer registries. Epidemiol Prev 2001;25:347-53.

22 Sant M, Capocaccia R, Badioni V, et al. Survival for retinoblastoma in Europe. Eur J Cancer 2001;37:730-5.

23 Saw SM, Tan N, Lee SB, et al. Incidence and survival characteristics of retinoblastoma in Singapore from 1968-1995. J Pediatr Ophthalmol Strabismus 2000;37:87-93.

24 Sanders BM, Draper GJ, Kingston JE. Retinoblastoma in Great Britain 1969-80: incidence, treatment, and survival. Br J Ophthalmol 1988;72:576-83 